\title{
Landwater variation in four major river basins of the Indochina peninsula as revealed by GRACE
}

\author{
Keiko Yamamoto ${ }^{1}$, Yoichi Fukuda ${ }^{1}$, Toshiyuki Nakaegawa ${ }^{2}$, and Jun Nishijima ${ }^{3}$ \\ ${ }^{1}$ Department of Geophysics, Graduate School of Science, Kyoto University, Kitashirakawa Oiwake-cho, Sakyo-ku, Kyoto, Japan \\ ${ }^{2}$ Climate Research Department, Meteorological Research Institute, 1-1 Nagamine, Tsukuba, Ibaraki, Japan \\ ${ }^{3}$ Department of Earth Resources Engineering, Graduate School of Engineering, Kyushu University, 6-10-1 Hakozaki, Higashi-ku, Fukuoka, Japan
}

(Received April 20, 2006; Revised September 4, 2006; Accepted November 6, 2006; Online published May 7, 2007)

\begin{abstract}
We estimated mass variations in four major river basins- the Mekong, Irrawaddy, Salween and Chao Phraya river basins - of the Indochina Peninsula using the newly released GRACE (Gravity Recovery and Climate Experiment) monthly gravity field solutions of UTCSR RL02 (University of Texas at Austin, Center for Space Research Release 02), JPL RL02 (Jet Propulsion Laboratory Release 02) and GFZ RL03 (GeoForschungsZentrum Potsdam Release 03). The estimated variations were compared with that calculated from a numerical model. The results show that there is a good agreement between the GRACE estimations and the model calculation for the Mekong and Irrawaddy basins, while the aggreement for the Salween and Chao Phraya basins is poor, mainly due to the spatial scale of the areas concerned. The comparison over the combined area of the four river basins shows fairly good agreement, although there are small quantitative discrepancies. The amplitudes of the annual signals of the GRACE solutions are 0.9- to 1.4-fold larger than that of the hydrological model, and the phases are delayed about 1 month compared with the model signal. The phase differences are probably due to improper treatments of the groundwater storage process in the hydrological model, suggesting that the GRACE data possibly provide constraints to the model parameters.
\end{abstract}

Key words: GRACE, satellite gravity, gravity variations, water storage variations, precise gravity measurements.

\section{Introduction}

Since the successful launch of GRACE (Gravity Recovery and Climate Experiment) in March 2002, monthly global gravity field variations have been provided with an unprecedented accuracy as a series of spherical harmonic coefficients (Tapley et al., 2004a). The variations in the temporal gravity field revealed by GRACE, which can be interpreted as surface mass changes, have been widely employed for studies of landwater movements (Tapley et al., 2004b), ocean flow (Chambers et al., 2004), ice sheet mass changes (Velicogna and Wahr, 2006), and mass changes associated with an earthquake (Han et al., 2006). Among these applications, one of the most promising is the monitoring of landwater movements or terrestrial water storage, and several studies have already revealed that GRACE data can be applicable to monitoring regional land water mass variations at spatial scale greater than $1000 \mathrm{~km}$ (see, Wahr et al., 2004; Chen et al., 2005). However, it is not yet clear whether GRACE can reveal variations with a smaller spatial scale, and more case studies are necessary to confirm the applicability of GRACE data.

We have been planning to conduct precise in-situ gravity measurements combined with GPS and groundwater level measurements for monitoring local or urban scale groundwater variations in urbanized cities of East Asia (Research

Copyright (c) The Society of Geomagnetism and Earth, Planetary and Space Sciences (SGEPSS); The Seismological Society of Japan; The Volcanological Society of Japan; The Geodetic Society of Japan; The Japanese Society for Planetary Sciences; TERRAPUB
Institute for Humanity and Nature, 2005). In this project, we have selected Bangkok in Thailand as one of the research targets. Bangkok is located at the western part of the Indochina Peninsula where large annual and interannual landwater variations due to the Southeast Asian Monsoon climate are traditionally expected. These variations may cause gravity changes on the Earth's surface, and these influences, at least conceptually, should be removed from the in-situ gravity measurement data. Furthermore, we consider that an accurate knowledge of regional or relatively largescale landwater variations is indispensable for a better understanding of urban-scale variations.

In line with these points of views, we decided to employ GRACE data for estimating regional scale mass variations in the Indochina Peninsula. As such, to investigate the applicability of GRACE data, we attempted to recover the mass variations associated with four major river basins of the Indochina Peninsula - the Mekong, Irrawaddy, Salween and Chao Phraya river basins. Figure 1 shows the location of these river basins. The combined area of these four basins is $1,750,000 \mathrm{~km}^{2}$, which is probably large enough for GRACE to recover the mass variations, while the square measures of the Mekong, Irrawaddy, Salween and Chao Phraya river basins are 814,000, 425,000, 330,000 and $178,000 \mathrm{~km}^{2}$, respectively. The most interesting question is therefore 'Which mass variations can be revealed by GRACE data?'.

In this study, we employed newly released GRACE data sets from three data centers-the University of Texas at 
Table 1. Availability of the newly released GRACE Level 2 (near) monthly gravity field solutions of UTCSR RL02, JPL RL02 and GFZ RL03.

\begin{tabular}{|c|c|c|c|c|c|c|c|c|c|c|c|c|}
\hline Year & Jan & Feb & Mar & Apr & May & Jun & Jul & Aug & Sep & Oct & Nov & Dec \\
\hline 2003 & $\bullet$ & $\odot \odot$ & $\odot \odot$ & $\circ \odot$ & $\circ \odot$ & & $\odot \odot$ & 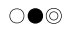 & $\circ \odot$ & $\bullet \bullet$ & $\circ \odot$ & 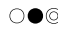 \\
\hline 2004 & $\bullet$ & $\odot$ & $\odot \odot$ & $\odot \bullet$ & $\odot \odot$ & ○(-) & & & & & $\bigcirc \odot$ & 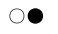 \\
\hline 2005 & $\circ \bullet$ & $\odot \odot$ & $\circ \odot$ & 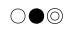 & $\bullet$ & $\bullet$ & - & - () & () & () & () & \\
\hline
\end{tabular}

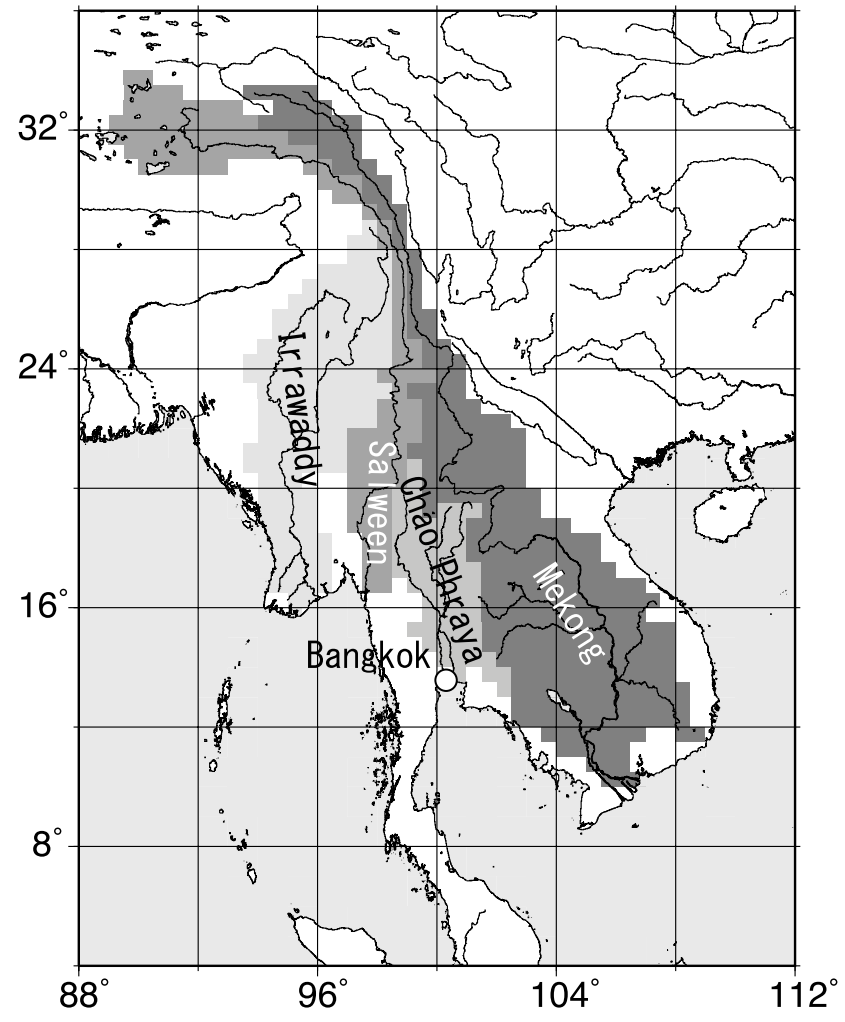

Fig. 1. Locations of the drainage areas of Mekong, Irrawaddy, Salween and Chao Phraya river basins.

Austin, Center for Space Research Release 02 (UTCSR RL02; Bettadpur, 2005), GeoForschungsZentrum Potsdam Release 03 (GFZ RL03; Flechtner, 2005a) and Jet Propulsion Laboratory Release 02 (JPL RL02; Watkins, 2005). The background gravity models and the $C_{20}$ values of these three data sets have been significantly upgraded compared with the previous version (UTCSR Release 01). In addition, the ocean tide and the short-period ocean models employed for the corrections have been also revised in the UTCSR RL02 and GFZ RL03 data sets. Using these data sets, we estimate here the regional scale mass variations and discuss several error sources as well as compare the results.

\section{Data Processing}

\subsection{Data sets}

We employed (near) monthly gravity field solutions up to full degree and order 120 from UTCSR RL02, JPL RL02 and GFZ RL03 GRACE Level 2 data sets. The data periods are summarized in Table 1 , and we employed all of the data sets from 2003 to 2005 . The numbers of the data sets are 22 (UTCSR RL02), 27 (JPL RL02) and 27 (GFZ RL03). Variable components of the gravity field solutions were calculated by subtracting the average value over the whole periods from the monthly solutions.

For comparison and verification of the estimated mass variations, we employed a combined mass variation model that consisted of the following ocean and landwater models: (1) the ocean bottom pressure data of ECCO (Estimating the Circulation and Climate of the Ocean)_JPL Ocean Data Assimilation Project model of Kalman filter run with data assimilated with respect to the control mean (kf049f; Fukumori et al., 1999); (2) the landwater model by the Japan Meteorological Agency, which is a combined model of the Simple Biosphere (SiB) model (Sellers et al., 1988) and Global River flow for TRIP (GRiveT) model (Hosaka et al., 2006; Nohara et al., 2006). The landwater model takes into consideration the variations in snow storage, soil moisture (down to $1 \mathrm{~m}$ from the surface) and river storage, while the groundwater storage process is only considered insufficiently. The combined model data were averaged over time corresponding to the GRACE solution periods (nearly monthly), and variable components were calculated by subtracting the average of the whole period. The components of $C_{00}, C_{10}, C_{11}$ and $S_{11}$ were removed from the model coefficients for consistency with the GRACE data.

\subsection{Recovery of the regional mass variations}

For the recovery of regional mass variations, Swenson and Wahr (2002) developed a method in which they designed a regional spatial filter so as to minimize both the satellite measurement error and the leakage error (unwanted signals from nearby regions). Swenson et al. (2003) revised the method to optimize it for signals expected in actual GRACE data. In this study, we followed the modified method of Swenson et al. (2003).

The surface mass variability over the region $\Delta \sigma_{\text {region }}$ was approximated by the following equations:

$$
\begin{aligned}
\Delta \sigma_{\text {region }}= & \sum_{l=0}^{l \max } \sum_{m=0}^{l} \frac{1}{\Omega_{\text {region }}} \frac{a \rho_{E}}{3} \frac{(2 l+1)}{\left(1+k_{l}\right)} \\
& \times\left(W_{l m}^{C} \Delta \bar{C}_{l m}+W_{l m}^{S} \Delta \bar{S}_{l m}\right),
\end{aligned}
$$

where $\Omega_{\text {region }}$ is the angular area of the region, $a$ is the equatorial radius, $\rho_{E}$ is the average density of the Earth, $k_{l}$ is the load Love number of degree $l, W_{l m}^{C}$ and $W_{l m}^{S}$ are the designed filter coefficients, $\Delta \bar{C}_{l m}$ and $\Delta \bar{S}_{l m}$ are fully normalized Stokes coefficients of degree $l$ and order $m$ and $l_{\max }$ is the truncated degree of the spherical harmonics.

$W_{l m}^{C}$ and $W_{l m}^{S}$ are obtained by the following equation

$$
\left\{\begin{array}{l}
W_{l m}^{C} \\
W_{l m}^{S}
\end{array}\right\}=\left[1+\frac{2(2 l+1) B_{l}^{2}}{\sigma_{0}^{2} G_{l}\left(1+k_{l}\right)^{2}}\left(\frac{a \rho_{E}}{3}\right)^{2}\right]^{-1}\left\{\begin{array}{c}
\vartheta_{l m}^{C} \\
\vartheta_{l m}^{S}
\end{array}\right\}
$$

where $B_{l}$ is degree amplitudes of the satellite measurement errors, $\sigma_{0}^{2}$ is the local signal variance, $G_{l}$ is the Legendre coefficients of a covariance function and $\vartheta_{l m}^{C}$ and $\vartheta_{l m}^{S}$ are 


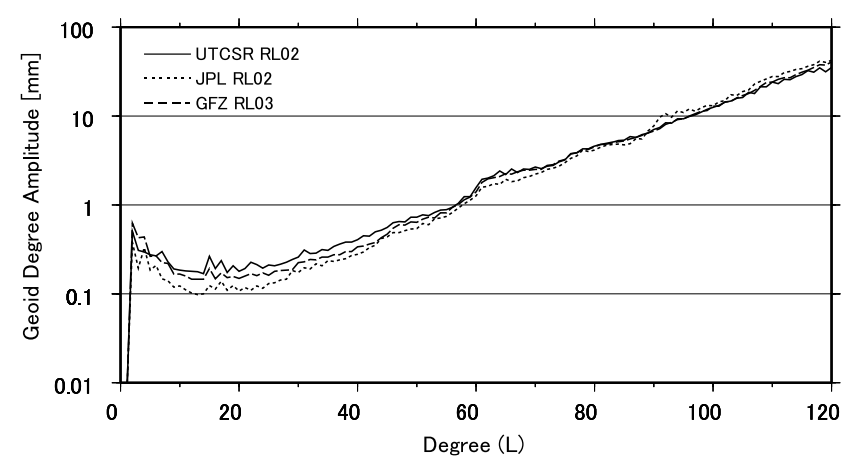

Fig. 2. Degree amplitudes of the estimated error of UTCSR RL02, JPL RL02 and GFZ RL03 data sets.

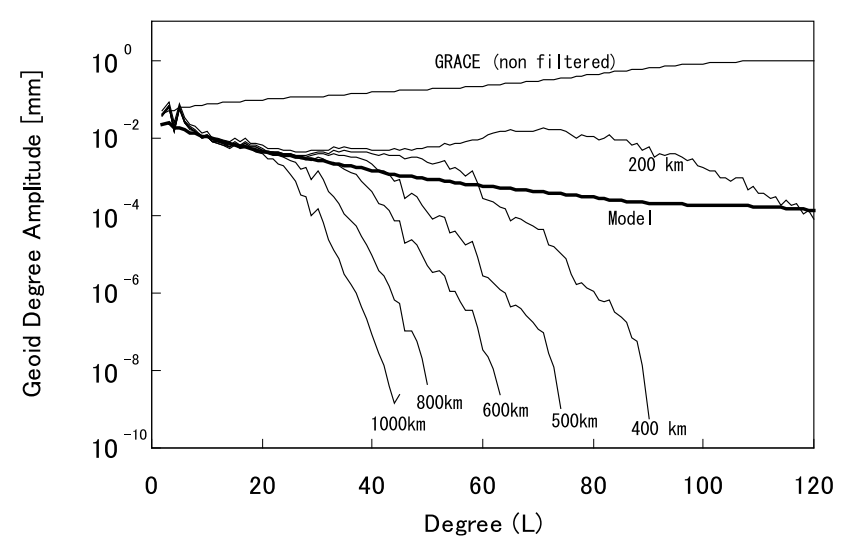

Fig. 3. Degree amplitudes for the combined river area of the four rivers calculated from the filtered GRACE data with different correlation lengths (from 200 to $1000 \mathrm{~km}$ ). Degree amplitudes of non-filtered GRACE data and the landwater model are also shown.

the spherical harmonic coefficients of the regional template, i.e., 1 inside and 0 outside the area concerned.

To suppress the higher degrees errors effectively, we employed the Gaussian covariance function

$$
G(\gamma, d)=\exp \left[\frac{-(1-\cos \gamma) \ln 2}{(1-\cos (d / a))}\right],
$$

as the covariance function, where $\gamma$ is angular distance and $d$ is correlation length.

For the optimal design of the regional filter, $B_{l}$ and $d$ should be fixed in advance, and $\sigma_{0}^{2}$ is determined so as to minimize the sum of the satellite measurement errors and the leakage errors iteratively. Finally, the amplitude degradation was corrected by multiplying a factor which was given as the ratio between non-filtered and filtered model data.

To determine the $B_{l}$ values, we have to know the error degree amplitudes of the GRACE measurements. However, these values are not available at present. As an approximation of the error degree amplitudes, Wahr et al. (2004) employed a value 1.1-fold as large as the residual signals which were obtained by removing constant and annual components. We adopted the same approximation to evaluate the error degree amplitudes. Figure 2 shows the estimated error degree amplitudes of the three data sets.

Using a hydrological model, Swenson et al. (2003) investigated the $d$ dependence of the recovered signal and re-

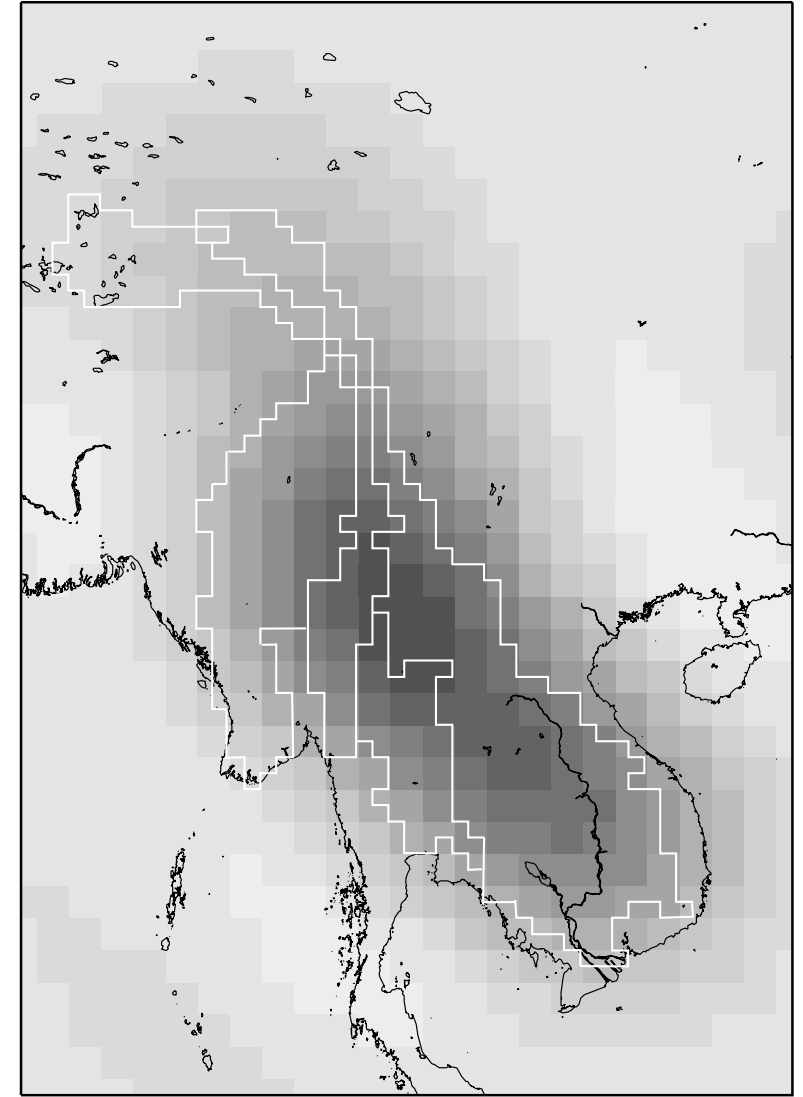

\section{$\begin{array}{llllllllll}-8 & -4 & 0 & 4 & 8 & 12 & 16 & 20 & 24 & 28\end{array}$}

Fig. 4. An optimal filter for the JPL RL02 data sets designed for the combined area of the Mekong, Irrawaddy, Salween and Chao Phraya river basins. The white lines show the boundaries of the drainage areas.

ported that the optimal $d$ value was between 200 and 800 $\mathrm{km}$ in most cases. This range of spatial scale is consistent with the correlation length of typical soil moisture variations. Chen et al. (2005a) reported that GRACE results correspond well to landwater model estimations across the world when the Gaussian filter with $d=800 \mathrm{~km}$ was applied. In this study, to find an appropriate $d$ value, we compared degree amplitudes of a hydrological model inside the area covered by the filtered GRACE data by applying Eq. (2) to the Stokes coefficients.

Figure 3 shows the degree amplitudes of the filtered (corresponding to different $d$ values from 200 to $1000 \mathrm{~km}$ ) and non-filtered GRACE data (JPL RL02) together with the hydrological model degree amplitudes for the combined area of the four rivers. Note that almost the same results were obtained even if UTCSR RL02 or GFZ RL03 data were employed. Although a smaller $d$ value is beneficial for detecting fine spatial scale mass variations, it suffers from measurement errors at higher degrees. Conversely, a larger $d$ value can suppress the higher degree errors, but it loses spatial resolution; further, widespread leakage errors prevent accurate mass estimation. Weighing the trade-off, we selected $600 \mathrm{~km}$ as the $d$ value from Fig. 3. We used the 
(a)

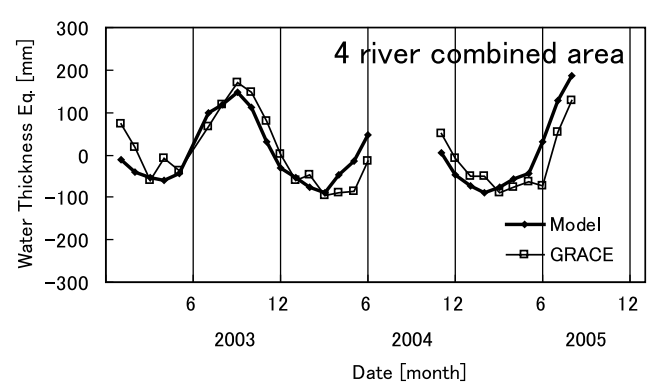

(c)

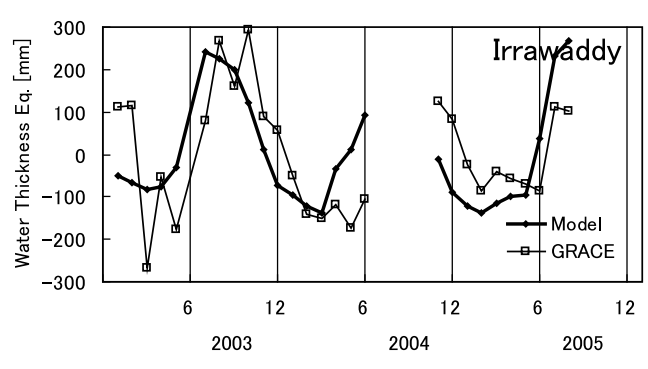

(e) (b)

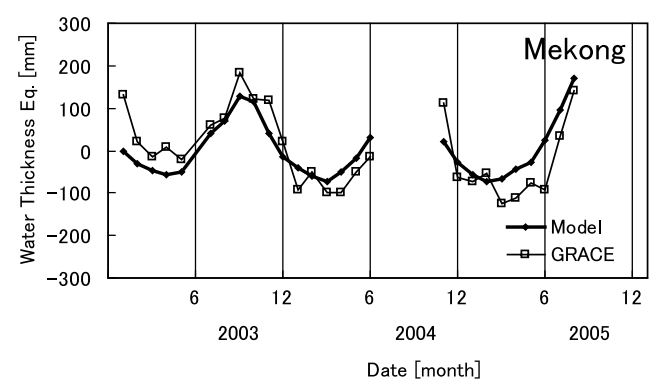

(d)

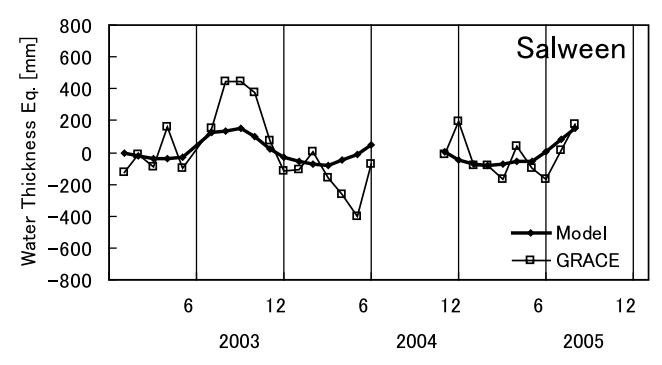

Date [month]

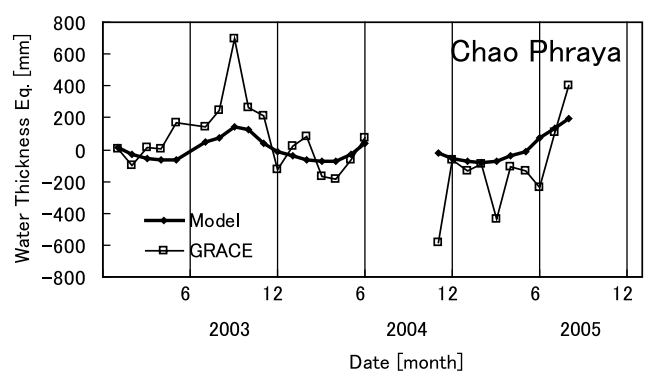

Fig. 5. Estimated mass variations for: (a) the combined area of the four rivers, and the (b) Mekong, (c) Irrawaddy, (d) Salween, and (e) Chao Phraya river basins. The JPL RL 02 data set and correlation length $d=600 \mathrm{~km}$ are used. The mass variations estimated from the model are also shown. Note that the vertical scales of (d) and (e) are different from the others.

same $d$ value throughout this study for all of the test areas because the correlation length of landwater signals and the GRACE measurement errors should be almost the same in the neighboring areas. As an example, Fig. 4 shows the designed filter for the combined area of the four rivers.

After applying the filters, we calculated the surface mass variations using Eq. (1). Because the degree 0 and degree 1 terms were not included in the GRACE Level 2 products, these terms were omitted in the calculation.

\subsection{Error estimations}

Because the regional filter is optimized by the trade-off between the satellite measurement error and the leakage error, it is impossible to reduce these errors simultaneously. The satellite measurement errors, which are shown as the error bars in the following figures, were calculated using the following equation:

$$
\begin{aligned}
& \Delta \varepsilon_{\text {region }}=\frac{1}{\Omega_{\text {region }}} \\
& \times \sqrt{\sum_{l=0}^{l \max }\left(\frac{a \rho_{E}}{3} \frac{(2 l+1)}{\left(1+k_{l}\right)}\right)^{2} \sum_{m=0}^{l}\left[\left(W_{l m}^{C} \Delta \bar{C}_{l m(\text { error })}\right)^{2}+\left(W_{l m}^{S} \Delta \bar{S}_{l m(\text { error })}\right)^{2}\right]} .
\end{aligned}
$$

Here, the same regional filters are applied to the GRACE error coefficients.

The leakage errors were estimated as follows. We first calculated the Stokes coefficients associated with the leakage effects using Eq. (5) by integrating $\Delta \sigma(\theta, \lambda)$ only outside the area concerned

$$
\begin{aligned}
\left\{\begin{array}{c}
\Delta \bar{C}_{l m} \\
\Delta \bar{S}_{l m}
\end{array}\right\}= & \frac{3\left(1+k_{l}\right)}{4 \pi a \rho_{E}(2 l+1)} \iint \Delta \sigma(\theta, \lambda) \bar{P}_{l m}(\cos \theta) \\
& \times\left\{\begin{array}{c}
\cos (m \lambda) \\
\sin (m \lambda)
\end{array}\right\} \sin \theta d \theta d \lambda
\end{aligned}
$$

where $\Delta \sigma(\theta, \lambda)$ is the surface density at colatitude $\theta$ and longitude $\lambda, \bar{P}_{l m}(\cos \theta)$ is the fully normalized Legendre function of degree $l$ and order $m$. The leakage effects were then estimated by applying the same filter designed in Section 2.2 to the derived Stokes coefficients. Finally, the effects were subtracted from the GRACE gravity solutions.

There may be two candidates for the input data of $\Delta \sigma(\theta, \lambda)$ in Eq. (5): one is calculated from model values and the other from GRACE data. As discussed in the following sections, we tested both cases and ultimately chose to use the GRACE data in this study. Note that to suppress the large errors of higher degree coefficients which 


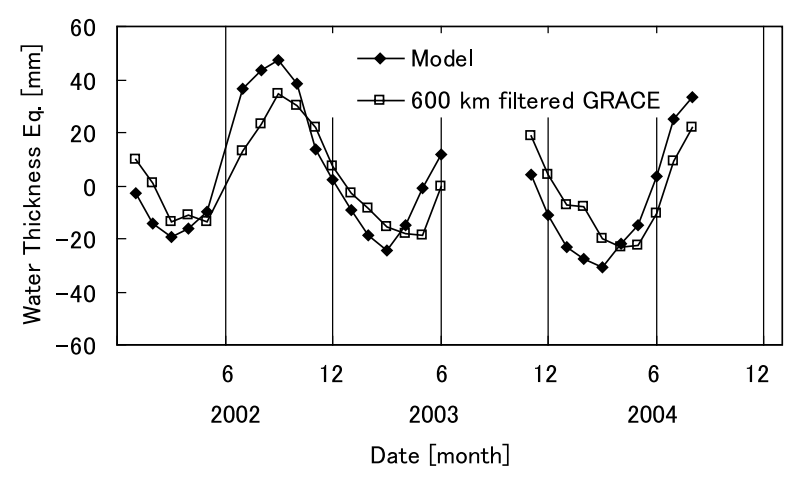

Fig. 6. Comparison of the leakage estimations. For the estimations, the model and the 600-km global filtered GRACE data are employed as the surface mass distributions.

may cause an inaccurate estimation of the leakage errors, we applied the globally normalized Gaussian filter (Wahr et al., 1998) with the same $d$ value to the original GRACE data.

\section{Results and Discussion}

Although most parts of following discussion are based on the JPL RL02 results, the results obtained by the other data sets are essentially the same. The comparisons between the three data sets are discussed in Sections 3 and 4.

\subsection{Estimation of the regional mass variations}

Figure 5 shows the estimated mass variations from GRACE. The model values, which are the regional averages of the gird values, are also shown in Fig. 5.

The GRACE estimations of the combined area of the four river basins (Fig. 5(a)) and the Mekong river basin (Fig. 5(b)) are in good agreement with the model values. The estimated values for the Irrawaddy river basin (Fig. 5 (c)) basically agree with the model values, although the GRACE estimation in Fig. 5(c) appears to be slightly noisier compared with Fig. 5(a) and (b).

Because the widths of the Salween and Chao Phraya river basins are smaller than the correlation length $d$ of 600 $\mathrm{km}$, a smaller $d$ might be preferable for these areas if the measurement errors were small enough. However, as shown in Fig. 5(d) and (e), the errors were larger than the derived mass variations, and the results appear to be unrealistic. If we used smaller $d$ values, the errors became much larger due to higher degree errors.

In addition to the size of the basin, its shape is another important factor for the precise mass recovery. The error distribution of the GRACE monthly gravity field solutions is not isotropic, but is strongly correlated with the satellite track, i.e., the north-south direction. These errors are called "striping" (e.g. Swenson and Wahr, 2006). The striping error is more serious for the extremely north-southoriented basins like Salween than for the isotropic or eastwest-oriented basins. This is another reason why the mass recovery in the Salween and Chao Phraya river basins are quite difficult.

In the following discussion, we only refer to the results on the combined area of the four rivers, but the same discussions are basically valid for the cases of the Mekong and Irrawaddy river basins. (a)

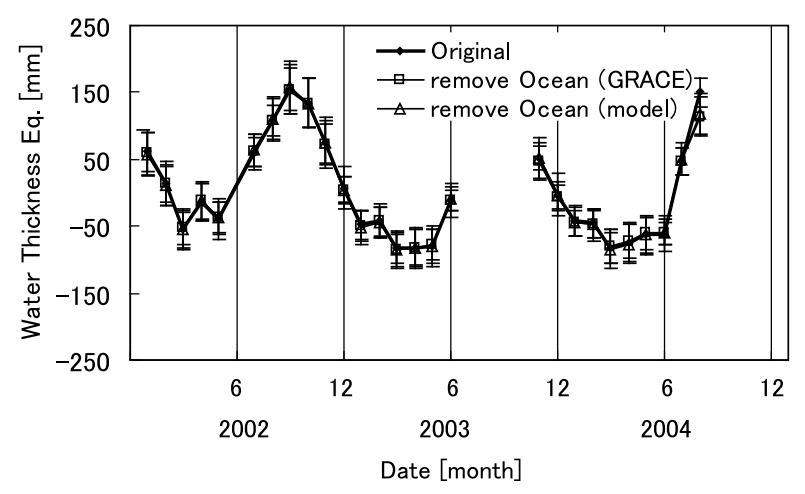

(b)

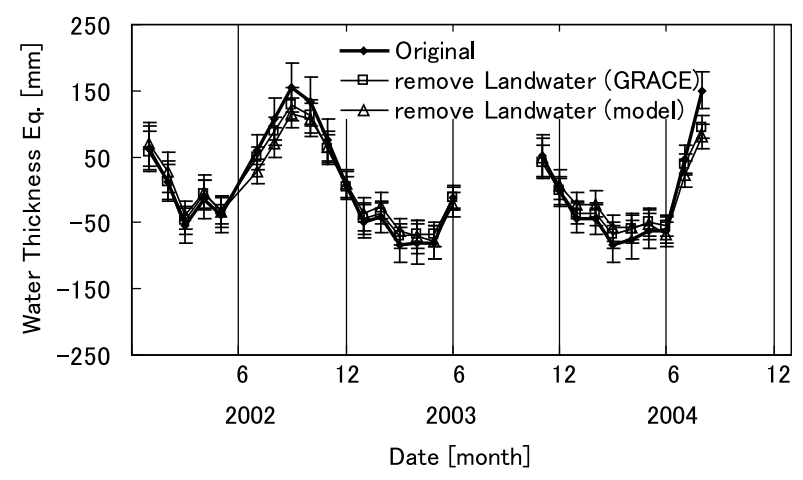

Fig. 7. Comparison of the mass variations before and after corrections. (a) The leakage influence of the ocean, (b) the leakage influence of the landwater. The leakage influence is estimated using the GRACE data and the model data.

\subsection{Leakage effect}

As previously discussed, we have two choices of surface mass densities for the estimation of the leakage effect. Figure 6 shows a comparison of these in which it is apparent there are slight differences between the two estimations in both amplitude and phase. One of the reasons for the amplitude difference may be the influence of the global filter applied to the GRACE data. However, at present, further discussions on the amplitudes are quite difficult due to the uncertainties of the model amplitude and the GRACE measurement errors.

On the other hand, it is true that the present landwater model has a problem in treating the groundwater storage and that it causes the error in phases. Because most of leakage errors come from the adjacent area where large groundwater signals are expected, the model error is more serious for the final results. Therefore, in this study, we ultimately decided to employ the GRACE data for estimating the leakage effects. Kanzow et al. (2005) reported that GRACE errors in the ocean area and on the land area are different. Therefore, we estimated the leakage effects from the ocean area and the land area separately. Figure 7 shows the results. In this figure, the estimations using model data are also plotted for the comparison. Compared with the previous data set (UTCSR RL01), which has larger errors in the ocean than on land (Kanzow et al., 2005), the short period ocean model is updated in the newly released data sets 
(a)

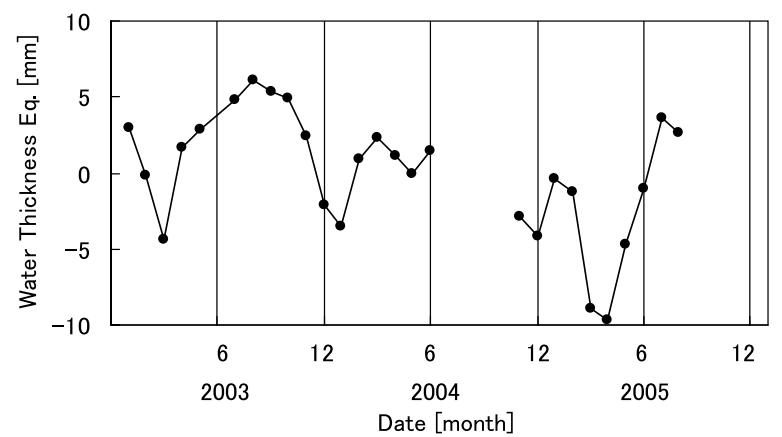

(b)

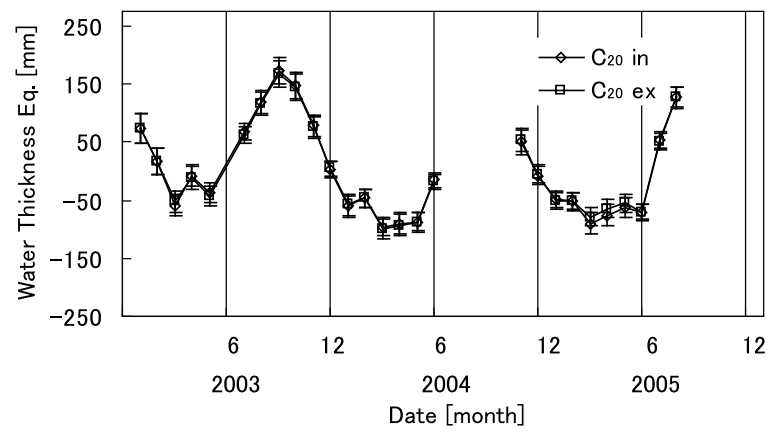

Fig. 8. (a) Contribution of the $C_{20}$ value to the mass variations in the combined area of the four rivers. (b) Estimated total mass variations, including and excluding the $C_{20}$ value.

(Flechtner, 2005b). However, because the ocean effect is very small, as shown in Fig. 7(a), the improvement does not affect our mass estimation, and the errors due to the ocean models can be completely negligible in this area. As shown in Fig. 7(b), the leakage effect of the landwater is larger than that of the ocean. The difference between the results using the GRACE data and the model data is small, and it can be safely neglected.

\subsection{Effect of $C_{20}$}

Chen et al. (2005b) reported that the $C_{20}, C_{21}$ and $S_{21}$ values of the previous version (UTCSR RL01) are inaccurate compared to the ones derived from Earth rotation (EOP) and satellite laser ranging (SLR) observations. They also reported that the replacement of the degree 2 values by those of EOP/SLR improved the estimation results in several large river basins and that fairly good agreements with the landwater model were obtained. Inaccurate $C_{21}$ and $S_{21}$ values of the previous version came from the absence of the ocean pole tide correction and could, therefore, be improved by the proper correction. On the other hand, the $C_{20}$ value cannot be well determined from GRACE L-L SST data mainly due to the orbit geometry and the separation length between the two satellites. Therefore, most of the previous studies excluded the $C_{20}$ value in the estimation of mass variations (e.g. Wahr et al., 2004).

In the newly released data sets, the ocean pole tide has already been corrected. It has also been announced that the accuracies of the $C_{20}$ values have been dramatically improved. To confirm the improvement of the $C_{20}$ values, we calculated the $C_{20}$ contributions separately. Figure 8(a) shows the results. The seasonal variation of the $C_{20}$ con- (a)

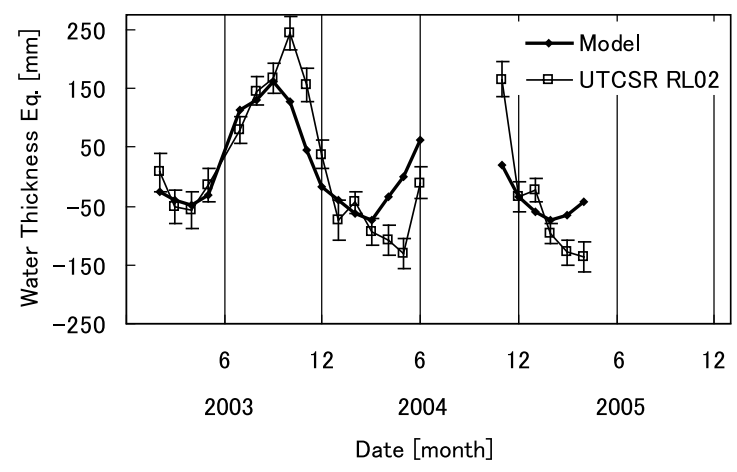

(b)

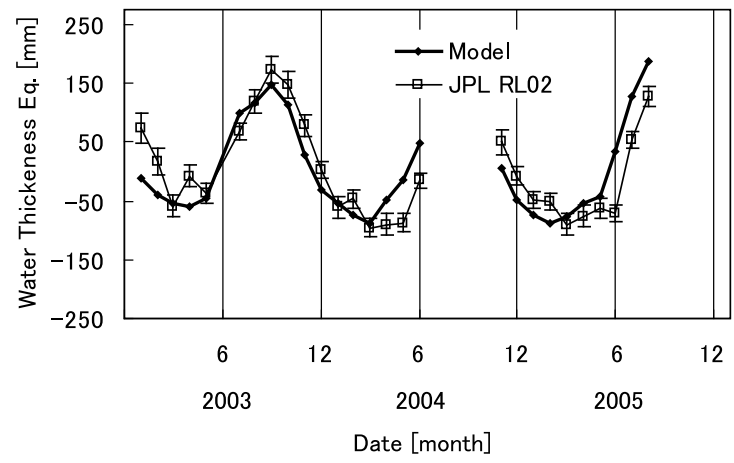

(c)

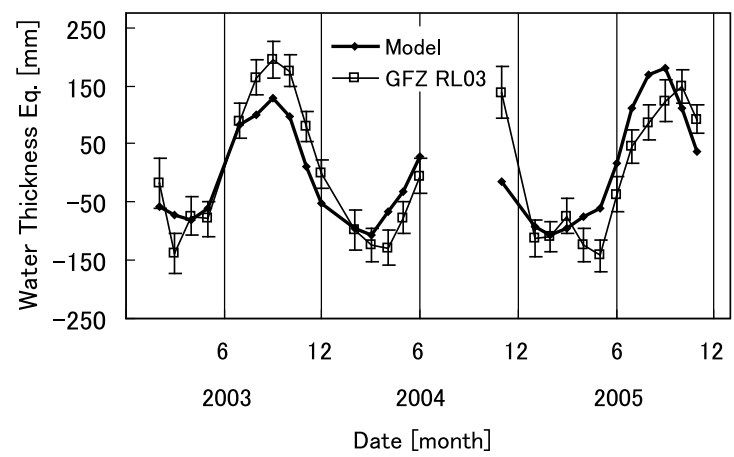

Fig. 9. Mass variations in the combined area of the four rivers recovered by the (a) UTCSR RL02, (b) JPL RL02, and (c) GFZ RL03 data sets. The estimated variations by the model are also shown in the figures.

tributions is slightly different from the total mass variation; in fact, the $C_{20}$ values of the three data setst still have relatively large differences of about $10 \mathrm{~mm}$-equivalent water thickness at most (the data are not shown). However, as shown in Fig. 8(b), the influence of $C_{20}$ on the final result is only about $10 \%$ of the total signal at most, and there is no positive reason to exclude the $C_{20}$ values. Therefore, we decided to include thesevalue in the estimations.

\subsection{Comparison of the results of the 3 data sets}

Figure 9 shows the derived mass variation in the combined area of the four rivers based on the three new data sets. A common feature to all three data sets is a good agreement with the model in 2003 and the first half of 2004. Large discrepancies, especially in UTCSR RL02 and GFZ RL03, are seen in November 2004, just after the GRACE data gap from July to October 2004, when the solutions are unstable because of the repeat orbit of the GRACE satellites (Wag- 
Table 2. The annual amplitudes and the phases lags (+delay) of the derived mass variations relative to those estimated from the model data. Two cases of using all data and excluding the data of November 2004 are shown.

\begin{tabular}{lcccc}
\hline & \multicolumn{2}{c}{ All Data } & \multicolumn{2}{c}{ Excluding Nov. 2004 } \\
& Amplitude & Phase lag [day] & Amplitude & Phase lag [day] \\
\hline UTCSR RL02 & 1.37 & +29.5 & 1.32 & +26.7 \\
JPL RL02 & 0.92 & +27.4 & 0.94 & +26.7 \\
GFZ RL03 & 1.18 & +24.4 & 1.15 & +20.8 \\
\hline
\end{tabular}

ner et al., 2006; Yamamoto et al., 2005). In this period, the resolution of the GRACE monthly field is significantly degraded to only about degree 30 (Wagner et al., 2006). We suspect that there may be a GRACE data error after the data gap period.

In any case, all three results show clear seasonal mass variations even though slight differences exist, especially in the amplitudes. The amplitude of the estimated mass variation is large and is in the order of UTCSR RL02, GFZ RL03 and JPL RL02. This corresponds to the order of the degree powers of the variable components, as shown in Fig. 10. These differences may be due to the different data processing scheme of each data center, namely the differences in GPS data processing and the background gravity field model employed. Although we cannot conclude which data set is the most preferable and/or reliable at present, Fig. 9 shows that the best agreement between JPL RL02 and the model can be seen in this area.

\subsection{Annual variations}

Annual signals of the estimated mass variations were calculated for both GRACE and the model data sets and compared with each other. The signal fittings were conducted for two cases: one in which all the data were used and one in which the data of November 2004 were excluded. The results are summarized in Table 2.

Due to several uncertainties, as previously mentioned, we dare not discuss the amplitudes here. On the other hand, the phases of all three GRACE results commonly show a delay of about 1 month or less compared to the model. Chen et al. (2005b) compared the mass variation derived from GRACE data to the GLDAS landwater model (Rodell et al., 2004) in several large river basins and also pointed out the phase delay in the GRACE data. These researchers considered that one of the possible reasons for this delay is the influence of groundwater flow, which is not considered in the GLDAS model. In our cases, the same reason can be considered because the phase lag of the groundwater storage is not considered in either the SiB or GRiveT model.

\section{Conclusion}

We confirmed that the newly released GRACE Level 2 data sets should be useful to detect-at the very minimum-Mekong and Irrawaddy river basin-scale mass variations. The relatively large-scale mass variation of the combined area of the four rivers, of which the spatial scale is larger than $1000 \mathrm{~km}$, shows a fairly good agreement with the numerical model. However, it is still difficult to reveal the mass variations in the spatial scale of the Salween basin or Chao Phraya basins. This is mainly because the insufficient spatial resolution of the current GRACE data sets, but another important issue is the non-isotropic feature of the GRACE errors. There may be some space for improving this feature by introducing such techniques as the non-isotropic filtering (Han et al., 2005) and/or de-striping (Swenson and Wahr, 2006).

With respect to the comparison of the results based on the data from the three different data centers, the obtained amplitudes are slightly different from each other. Although JPL RL02 shows the best agreement with the model, it is still difficult to conclude which data set is the most reliable because of the insufficient reliability of the model itself. On the other hand, the phases of the estimated mass variations of all three data sets commonly show a 20- to 30-day delay compared to the model. A possible reason is the groundwater behavior which is not properly treated in the model. This suggests that GRACE can provide useful information to improve the hydrological model.

As a part of HIUSE (Human Impacts on Urban Subsurface Environment) project (Research Institute for Humanity and Nature, 2005), we intend to employ a new technique of precise gravity measurements combined with GPS positioning for monitoring the groundwater changes in Bangkok, which is located at the downstream region of the Chao Phraya river basin. GRACE resolution at present may not be sufficiently high to reveal the mass variation of the Chao Phraya river basin, mainly due to its spatial scale and the shape of the basin. However, it was able toy detect the regional mass variation over the combined area of the four rivers, including the Chao Phraya basin. For the purpose of detecting groundwater variations by means of in-situ gravity measurements, the monitoring of the background variations due to relatively larger scale landwater variation is indispensable. We believe that GRACE data can be used for the purpose.

We expect that a reliable discussion of the groundwater variation can be possible by combining the GRACE data and in-situ measurements. We also expect that the in-situ measurements will provide a constraint to investigating the discrepancies between the GRACE solutions and the hydrological model previously described.

Acknowledgments. We used GRACE Science Team data in this research and would like gratefully acknowledge our appreciation to the GRACE project for providing the GRACE Level 2 monthly gravity field solutions. This research was partially supported by the project "Human Impacts on Urban Subsurface Environment" (Project Reader: Makoto Taniguchi), Research Institute for $\mathrm{Hu}-$ manity and Nature (RIHN) and Grant-in-Aid for the 21st Century COE Program (Kyoto University, G3). We would like to express our gratitude to two reviewers, Dr. Jiliani Chen and Dr. Hiroshi Munekane for their constructive comments which improved the paper very much.

\section{References}

Bettadpur, S., UTCSR Level-2 Processing Standards Document For Level2 Product Release 0002 (Draft, November 4, 2005), GRACE 327-742 (CSR-GR-03-03), 2005.

Chen, J. L., C. R. Wilson, J. S. Famiglietti, and M. Rodell, Spatial sensitivity of the Gravity Recovery and Climate Experiment (GRACE) time-variable gravity observations, J. Geophys. Res., 110, B08408, doi:10.1029/2004JB003536, 2005a.

Chen, J. L., M. Rodell, C. R. Wilson, and J. S. Famiglietti, Low degree spherical harmonic influences on Gravity Recovery and Climate Experiment (GRACE) water storage estimates, Geophys. Res. Lett., 32, 
L14405, doi:10.1029/2005GL022964, 2005b.

Chambers, D. P., J. Wahr, and R. S. Nerem, Preliminary observations of global ocean mass variations with GRACE, Geophys. Res. Lett., 31, L13310, doi:10.1029/2004GL020461, 2004.

Flechtner, F., GFZ Level-2 Processing Standards Document For Level-2 Product Release 0003 (Rev. 1.1, November 04, 2005), GRACE 327-743 (GR-GFZ-STD-001), 2005a.

Flechtner, F., AOD1B Product Description Document (Rev. 2.1, November 04, 2005), GRACE 327-750 (GR-GFZ-AOD-0001), $2005 \mathrm{~b}$.

Fukumori, I., R. Raghunath, L. Fu, and Y. Chao, Assimilation of TOPEX/POSEIDON data into a global ocean circulation model: How good are the results?, J. Geophys. Res., 104(C11), 25647-25666, doi:10. 1029/1999JC900193, 1999.

Han, S. C., C. K. Shum, C. Jekeli, C. Y. Kuo, C. Wilson, and K. W. Seo, Non-isotropic filtering of GRACE temporal gravity for geophysical signal enhancement, Geophys. J. Int., 163, 18-25, doi:10.1111/j.1365246X.2005.02756.x, 2005.

Han, S. C., C. K. Shum, M. Bevis, J. Chen, and C. Y. Kuo, Crustal Dilatation Observed by GRACE After the 2004 Sumatra-Andaman Earthquake, Science, 33, 658-662, 2006.

Hosaka, M., D. Nohara, T. Nakaegawa, and S. Yukimoto, MRI Global River flow model using TRIP, J. Meteor. Soc. Jpn., 2006 (to be submitted).

Kanzow, T., F. Flechtner, A. Chave, R. Schmidt, P. Schwitzer, and U. Send, Seasonal variation of ocean bottom pressure derived from Gravity $\mathrm{Re}$ covery and Climate Experiment (GRACE): Local variation and global patterns, J. Geophys. Res., 110, C09001, doi:10.1029/2004JC002772, 2005.

Nohara, D., A. Kitoh, M. Hosaka, and T. Oki, Impact of Climate Change on River Discharge Projected by Multimodel Ensemble, J. Hydromet., 7, 1076-1089,doi:10.1175/JHM531.1, 2006.

Research Institute for Humanity and Nature, Research Project 2-4, Human Impacts on Urban Subsurface Environments, http://www.chikyu.ac.jp/ USE/, 2005.

Rodell, M., P. R. Houser, U. Jambor, J. Gottschalck, K. Mitchell, C.-J. Meng, K. Arsenault, B. Cosgrove, J. Radakovich, M. Bosilovich, J. K. Entin, J. P. Walker, D. Lohmann, and D. Toll, The Global Land Data Assimilation System, Bull. Amer. Meteor. Soc., 85, 381-394, doi:10. 1175/BAMS-85-3-381, 2004.

Sellers, P. J., Y. Mintz, Y. C. Sud, and A. Dalcher, Simple Biosphere model (SiB) for use within general circulation model, J. Atmos. Sci., 43, 505-
$531,1988$.

Swenson, S. and J. Wahr, Methods for inferring regional surface-mass anomalies from Gravity Recovery and Climate Experiment (GRACE) measurements of time-variable gravity, J. Geophys. Res., 107(B9), 2193, doi:10.1029/2001JB000576, 2002.

Swenson, S., J. Wahr, and P. C. D. Milly, Estimated accuracies of regional water storage variations inferred from the Gravity Recovery and Climate Experiment (GRACE), Water Resour. Res., 39(8), 1223, doi:10.1029/ 2002WR001808, 2003.

Swenson, S. and J. Wahr, Post-processing removal of correlated errors in GRACE data, Geophys. Res. Lett., 33, L08402, doi:10.1029/ 2005GL025285, 2006.

Tapley, B. D., S. Bettadpur, M. Watkins, and C. Reigber, The Gravity Recovery and Climate Experiment: Mission overview and early results, Geophys. Res. Lett., 31, L09607, doi:10.1029/2004GL019920, 2004a.

Tapley, B. D., S. Bettadpur, J. C. Ries, P. F. Thompson, and M. M. Watkins, GRACE Measurements of Mass Variability in the Earth System, Science, 203, 503-505, 2004b.

Velicogna, I. and J. Wahr, Measurements of Time-Variable Gravity Show Mass Loss in Antarctica, Science, 311, 1754-1756, 2006.

Wagner, C., D. Mcado, J. Klokocnik, and J. Kostelecky, Degradation of Geopotential Recovery from Short Repeat Cycle Orbits: Application to GRACE Monthly Fields, J. Geod., 80, 94-103, doi:10.1007/s00190006-0036-x, 2006.

Wahr, J., M. Molenaar, and F. Bryan, Time variability of the Earth's gravity field: Hydrological and oceanic effects and their possible detection using GRACE, J. Geophys. Res., 103(B12), 30205-30229, doi:10.1029/ 98JB02844, 1998.

Wahr, J., S. Swenson, V. Zlotnicki, and I. Velicogna, Time-variable gravity from GRACE: First results, Geophys. Res. Lett., 31, L11501, doi:10. 1029/2004GL019779, 2004.

Watkins, M. M., JPL Level-2 Processing Standards Document For Level-2 Product Release 02, GRACE 327-744, v2.0, 2005.

Yamamoto, K., T. Otsubo, T. Kubo-oka, and Y. Fukuda, A simulation study of effects of GRACE orbit decay on the gravity field recovery, Earth Planets Space, 57, 291-295, 2005.

K. Yamamoto (e-mail: yamamoto@kugi.kyoto-u.ac.jp), Y. Fukuda, T. Nakaegawa, and J. Nishijima 